\title{
Chemical proteomics, an integrated research engine for exploring drug-target- phenotype interactions
}

\author{
Ho Jeong Kwon ${ }^{1 *}$ and Peter Karuso ${ }^{2^{*}}$
}

Chemical proteomics has emerged as an integrated research engine for the discovery of new drugs and the agnostic identification of drug targets. It involves the development and combination of chemical tools, bioorthogonal techniques, phenotypic screens, target identification and validation, mode of action and chemoinformatics that together provide robust and high-throughput workflows for exploring drug-targetphenotype relationships. In particular, the focus is on the application of small molecules in proteomics and genome-wide techniques for the rapid identification of drug targets.

The special issue of Proteome Science highlights the state-of-the-art for workflows and applications of chemical proteomics with 3 review articles and 2 original research papers contributed by the leading scientists in the field.

Savistski and co-workers summarize the recent developments in thermal proteome profiling (TPP) [1]. This technique relies on the thermal stabilization of a protein when it is bound to its ligand and the detection of this stabilization through changes in that protein's concentration compared to an identical experiment with no added drug. In this respect it is similar to the drug affinity responsive target stability (DARTS) assay, which measures the difference in protein hydrolysis by a nonspecific protease with varying concentrations of a ligand [2]. Both assays can be done in live cells, in which case TPP is called the cellular thermal shift assay (CETSA) [3]. Combining CETSA with quantitative proteomics (using hypobaric tagging of the tryptic digest), provides a powerful platform for the identification of drug targets without the need for labeling of the ligand. Such techniques are at the vanguard of label-free techniques for

\footnotetext{
*Correspondence: kwonhj@yonsei.ac.k; peter.karuso@mq.edu.au

${ }^{1}$ Department of Biotechnology, Yonsei University, 50 Yonsei-ro,

Seodaemun-gu, Seoul 120-749, South Korea

${ }^{2}$ Department of Chemistry and Biomolecular Sciences, Macquarie University, Sydney, NSW 2109, Australia
}

(c) The Author(s). 2018 Open Access This article is distributed under the terms of the Creative Commons Attribution 4.0 International License (http://creativecommons.org/licenses/by/4.0/), which permits unrestricted use, distribution, and reproduction in any medium, provided you give appropriate credit to the original author(s) and the source, provide a link to the Creative Commons license, and indicate if changes were made. The Creative Commons Public Domain Dedication waiver (http://creativecommons.org/publicdomain/zero/1.0/) applies to the data made available in this article, unless otherwise stated. review described variants of the general workflow with a focus on elucidation of drug-target interactions as a means to generate target hypotheses for hits from phenotypic screens. Because quantitative proteomics techniques have extended the scope of target identification to the whole proteome level, this approach is receiving attention as a strategy to discover new targets and an important method for cellular metabolic mapping. In this regard, TPP could provide a unique approach to unveiling thousands of cellular dynamic environments for drugs of interest.

Protein-protein and drug-protein interactions have been studied by a variety of techniques including affinity chromatography, peptide arrays, activity and affinitybased probes, drug Western and photoaffinity labeling [5]. In the next paper, Lee and co-workers comprehensively review recent progress in photoaffinity labeling (PAL) technologies, developed by Westheimer in 1962 [6], based on three different photocrosslinkers (PLs); diazirines, benzophenones and arylazides. In this outstanding review, research examples of each PL type are nicely provided against multiple different proteins of interests. This review also provides some important examples of quantitative proteomics in the study proteinprotein interactions (PPIs) including general principles and future perspectives. Given the important role of PAL in studying PPIs, this review is timely for the readers in chemical proteomics fields, who should be thinking about this technique for the study of drugprotein and natural product-protein interactions.

In the third review, Kim and co-workers provide a targeted review on the use of the tetrazine ligation reactions in chemical proteomics. Since Sharpless introduced the idea of bioorthogonal "click chemistry" in 2001 [7], there has been over 20 different such reactions identified so far. This article reviews one of those bio-orthogonal cycloaddition reactions; the $[4+2]$ inverse demand Diels-Alder cycloaddition (tetrazine ligation), 
highlighting the chemical features of this reaction in biology and summarizing the applications of tetrazine ligation for protein imagining and drug-target identification. The authors review the range of fluorophores available with tetrazine groups and their use in protein and small molecule labeling and compare this to $\mathrm{Cu}(\mathrm{I})$ and copper-free Huisgen ligations and the use of cleavable and photoaffinity-based linkers in probe design.

In addition to these three review articles, Thomas et al. examines the influence of specificity of bivalent kinase probes that contain either a $\mathrm{SH} 2$ or $\mathrm{SH} 3$ binder plus an ATP mimic. In this excellent example of the design, synthesis and application of dual functional chemical proteomics probes, the authors confirmed the affinity and selectivity of their bivalent inhibitors against $\mathrm{Abl}$ along with other off targets. They found bivalent inhibitor A-2 has improved affinity as well as selectivity over the original ATP competitive inhibitor, KAM. These results provide interesting molecular design strategy applicable to many other bivalent inhibitors/probes that could find utility in chemical proteomics. Finally, Karuso and Kwon presented their findings on the unbiased, genome wide identification of a human protein target for the new antibiotic Daptomycin (DAP). They used a phage display cDNA expression platform (reverse chemical proteomics) to screen selected human cancer cDNA libraries for human binding partners for DAP [8]. Using this approach they identified the ribosomal protein S19 (RPS19), to be most the most common binding partner for DAP in all the cell lines. They validated this finding with a number of in vitro experiments, to confirm a role for DAP-RPS19 interaction as a possible target for cancer therapy. This paper provides a nice case study of the utility of reverse chemical proteomics to explore new targets of drugs with biological relevance that is applicable for the iterative identification of new targets where their mechanisms or target(s) and completely unknown. These sorts of methods will become increasing important as the need to identify a preclinical drug's targets becomes increasing important for drug optimization, mode of action and ultimately FDA approval.

Collectively, the papers of this special issue devoted to chemical proteomics nicely highlights and exemplifies the applications of this emerging research field that promises to overcome the hurdle to identify protein targets of biologically active small molecules with unknown modes of action. Chemical proteomics is also applicable to the functional study of proteomes, understanding modes of action and for the development of novel drugs modulating novel targets. We hope you will enjoy this special issue and trust that these articles will stimulate further developments in the field.

\section{Acknowledgements}

We are grateful for the support from Chemical Proteomics Initiative of Asia Oceania Human Proteomics Organization (AOHUPO) on the special issue of chemical proteomics. This work was supported in part by ARC grant

DP130103281 to P. K. and H. J. K., NRF grants 2015K1A1A2028365,

2015M3A9C4076321 to H. J. K.

\section{Funding}

This work was supported in part by ARC grant DP130103281 to P. K. and H. J. K., NRF grants 2015K1A1A2028365, 2015M3A9C4076321 to H. J. K.

Availability of data and materials

Not applicable.

Authors' contributions

All authors read and approved the final manuscript.

Ethics approval and consent to participate

Not applicable.

Consent for publication

N/A.

Competing interests

HoJeong Kwon is co-Editor-in-Chief and Peter Karuso is an editorial board member for Proteome Science.

\section{Publisher's Note}

Springer Nature remains neutral with regard to jurisdictional claims in published maps and institutional affiliations.

Received: 11 December 2017 Accepted: 11 December 2017

Published online: 09 January 2018

References

1. Savitski MM, Reinhard FB, Franken H, Werner T, Savitski MF, Eberhard D, Martinez Molina D, Jafari R, Dovega RB, Klaeger S, et al. Tracking cancer drugs in living cells by thermal profiling of the proteome. Science. 2014;346: 1255784.

2. Lomenick B, Hao R, Jonai N, Chin RM, Aghajan M, Warburton S, Wang J, Wu RP, Gomez F, Loo JA, et al. Target identification using drug affinity responsive target stability (DARTS). P Natl Acad Sci USA. 2009;106:21984-9.

3. Martinez Molina D, Jafari R, Ignatushchenko M, Seki T, Larsson EA, Dan C, Sreekumar L, Cao Y, Nordlund P. Monitoring drug target engagement in cells and tissues using the cellular thermal shift assay. Science. 2013;341:84-7.

4. Chang J, Kim Y, Kwon HJ. Advances in identification and validation of protein targets of natural products without chemical modification. Nat Prod Rep. 2016;33:719-30.

5. Karuso P. Modern methods for the isolation of natural product receptors. In: Liu H-W, Mander L, editors. Comprehensive natural products II, vol. Volume 9. Oxford: Elsevier; 2010. p. 513-67.

6. Singh A, Thornton ER, Westheimer FH. The photolysis of diazoacetylchymotrypsin. J Biol Chem. 1962;237:3006-8.

7. Kolb HC, Finn MG, Sharpless KB. Click chemistry: diverse chemical function from a few good reactions. Angew Chem Int Ed. 2001;40:2004-21.

8. Piggott AM, Karuso P. Identifying the cellular targets of natural products using T7 phage display. Nat Prod Rep. 2016;33:626-36. 